Reeks MW, Swailes DC, Bragg A. Is the kinetic equation for turbulent gasparticle flows ill-posed? Physical Review E 2018, 97(2), 023104.

DOI link

https://doi.org/10.1103/PhysRevE.97.023104

ePrints link

http://eprint.ncl.ac.uk/246826

Date deposited

$14 / 03 / 2018$

Copyright

(C2018 American Physical Society. This is the authors' accepted manuscript of an article that has been published in its final definitive form by the American Physical Society, 2018. 


\title{
Is the kinetic equation for turbulent gas-particle flows ill-posed?
}

\author{
M. Reeks\#乎 and D.C. Swailes\#\# \\ School of Mechanical \&6 Systems Engineering \# and School of Maths, \\ Statistics 8 Physics ${ }^{\# \#, ~ N e w c a s t l e ~ U n i v e r s i t y, ~ N e w c a s t l e ~ u p o n ~ T y n e ~ N E 1 ~ 7 R U, ~ U K ~}$ \\ A. $B r a g g^{\dagger}$ \\ Department of Civil \& Environmental Engineering, Duke University, USA
}

(Dated: August 1, 2017)

\begin{abstract}
This paper is about well-posedness and realizability of the kinetic equation for gas-particle flows and its relationship to the Generalized Langevin Model (GLM) PDF equation. Previous analyses claim that this kinetic equation is ill-posed, that in particular it has the properties of a backward heat equation and as a consequence, its solutions will in the course of time exhibit finite-time singularities. We show that the analysis leading to this conclusion is fundamentally incorrect because it ignores the coupling between the phase space variables in the kinetic equation and the time and particle inertia dependence of the phase space diffusion tensor. This contributes an extra +ve diffusion that always outweighs the contribution from the-ve diffusion associated with the dispersion along one of the principal axes of the phase space diffusion tensor. This is confirmed by a numerical evaluation of analytic solutions of these $+v e$ and $-v e$ contributions to the particle diffusion coefficient along this principal axis. We also examine other erroneous claims and assumptions made in previous studies that demonstrate the apparent superiority of the GLM PDF approach over the kinetic approach. In so doing we have drawn attention to the limitations of the GLM approach which these studies have ignored or not properly considered, to give a more balanced appraisal of the benefits of both PDF approaches.
\end{abstract}

\section{INTRODUCTION}

The Probability Density Function (PDF) approach has proved very useful in studying the behavior of stochastic systems. Familiar examples of its usage occur in the study of Brownian Motion [2] and in the kinetic theory of gases 3 . In more recent times it has been used extensively by Pope and others to model turbulence [6] and turbulence related phenomena such as combustion 13 . and atmospheric dispersion 9. This paper is about its application to particle transport in turbulent gas-flows where it has been been developed and refined over a number of years by numerous authors. It has been successfully applied to a whole range of turbulent dispersed flow problems involving mixing and dispersion as well as particle collisions and clustering in a particle pair formulation of the approach. It has also formed a fundamental basis for dealing with complex flows in formulating the continuum equations and constitutive relations for the dispersed phase precisely analogous to the way the Maxwell Boltzmann equation has been used in the kinetic theory. It has become an established technique for studying dispersed flows so much so that the method and its numerous applications are the subject of a recent book 31 and the subject of a chapter in the recent Multiphase flow Handbook [23]

There are currently two PDF approaches that have been used extensively to describe the transport, mixing and collisions of small particles in turbulent gas flows.

\footnotetext{
* mike.reeks@ncl.ac.uk

$\dagger$ andrew.bragg@duke.edu
}

The first approach referred to as the kinetic approach, is based on a kinetic equation for the $\operatorname{PDF} p(\boldsymbol{x}, \boldsymbol{v}, t)$ of the particle position $\boldsymbol{x}$ and velocity $\boldsymbol{v}$ at time $t$. This equation based on a particle equation of motion involving the flow velocity along a particle trajectory derived from a Gaussian stochastic flow field. In the kinetic equation the particles' random motion arisng from this stochastic field is manifest as a diffusive flux which is a linear combination of gradient diffusion in both $\boldsymbol{x}$ and $\boldsymbol{v}$. Transient spatio-temporal structures in the turbulence give rise to an extra force due to clustering and preferential sweeping of particles [10].

In the second PDF approach an equation for the PDF $p(\boldsymbol{x}, \boldsymbol{v}, \boldsymbol{u}, t)$ is constructed, where $\boldsymbol{u}$ is the carrier flow velocity sampled along particle paths. Thus, unlike the kinetic approach, the flow velocity in this approach is retained in the particle phase space, and is described by a model evolution equation. In particular, this PDF model is based on a generalized Langevin model (GLM) (see Pope [6]) where the velocity of the underlying carrier flow measured along a particle trajectory is described by a generalized Langevin equation. As such the associated PDF equation is described by a Fokker-Planck equation. This GLM PDF equation has sometimes been inappropriately referred to as the dynamic PDF equation [12] implying that it is a more general PDF approach from which the kinetic equation can in general be derived. However it is important to appreciate the kinetic equation is not a standard Fokker-Planck equation, since it captures the non-Markovian features of the underlying flow velocities.

The problem of closure and the associated realiz- 
ability and well posedness of PDF equations are profoundly important in the study of stochastic equations. So despite the successful application of the kinetic equation to a whole range of problems, recent claims in the literature of ill-posedness and realizability of this equation are disturbing and a serious concern. The root cause of this concern is the non-positive definiteness of the diffusion tensor associated with the phase space diffusion flux. That in particular this tensor has both $+v e$ and $-v e$ eigenvalues implying that along the eigenvectors with a $-v e$ eigenvalue the particle dispersion exhibits the properties of a backward diffusion equation leading to solutions with finite time singularities. In fact Minier and Profeta 12 following a detailed analysis of the relative merits of the 2 PDF approaches, have concluded that the kinetic equation is ill-posed and therefore an invalid description of disperse two-phase flows (except in the limiting case for particles with large Stokes numbers when the kinetic equation reduces to a Fokker-Planck equation). This raises a number of issues and inconsistencies that we wish to examine and resolve:

1. The closure of the diffusive terms in the kinetic equation is exact for a Gaussian process for the aerodynamic driving forces in the particle equation of motion. Not withstanding any -ve eigenvalues, such dispersion processes are demonstrably forward rather than backward in time with statistical moments that monotonically increase rather than decrease with time. This behaviour is reflected in the analytic solutions of the kinetic equation for particle dispersion in shear flows in which the mean shear is linear and the turbulence is statistically homogeneous and stationary (see Hyland et al [7], Swailes and Darbyshire [4]). In these generic flows, there is exact correspondence of the analytical solution with a random walk simulation using a Lagrangian particle tracking approach, solving the individual particle equations of motion in the associated Gaussian random flow field. See as an example the illustration in Figure 1 .

2. In simple generic flows the GLM PDF equation is entirely consistent with the kinetic equation i.e the kinetic equation is recoverable from the GLM equations and has exactly the same solution for the same mean flows and statistical correlations for the turbulent velocity $\boldsymbol{u}$ along particle trajectories. They are both compatible with a Gaussian process. The claim of ill posedness of the kinetic equation would therefore seem to contradict the well posedness associated with the Fokker-Planck equation of the GLM.

So the first objective of the analysis we present here is to show that despite the non positive definiteness of the phase space diffusion tensor, this does not imply backward diffusion and the existence of finite time singularities, that the kinetic equation is well posed and
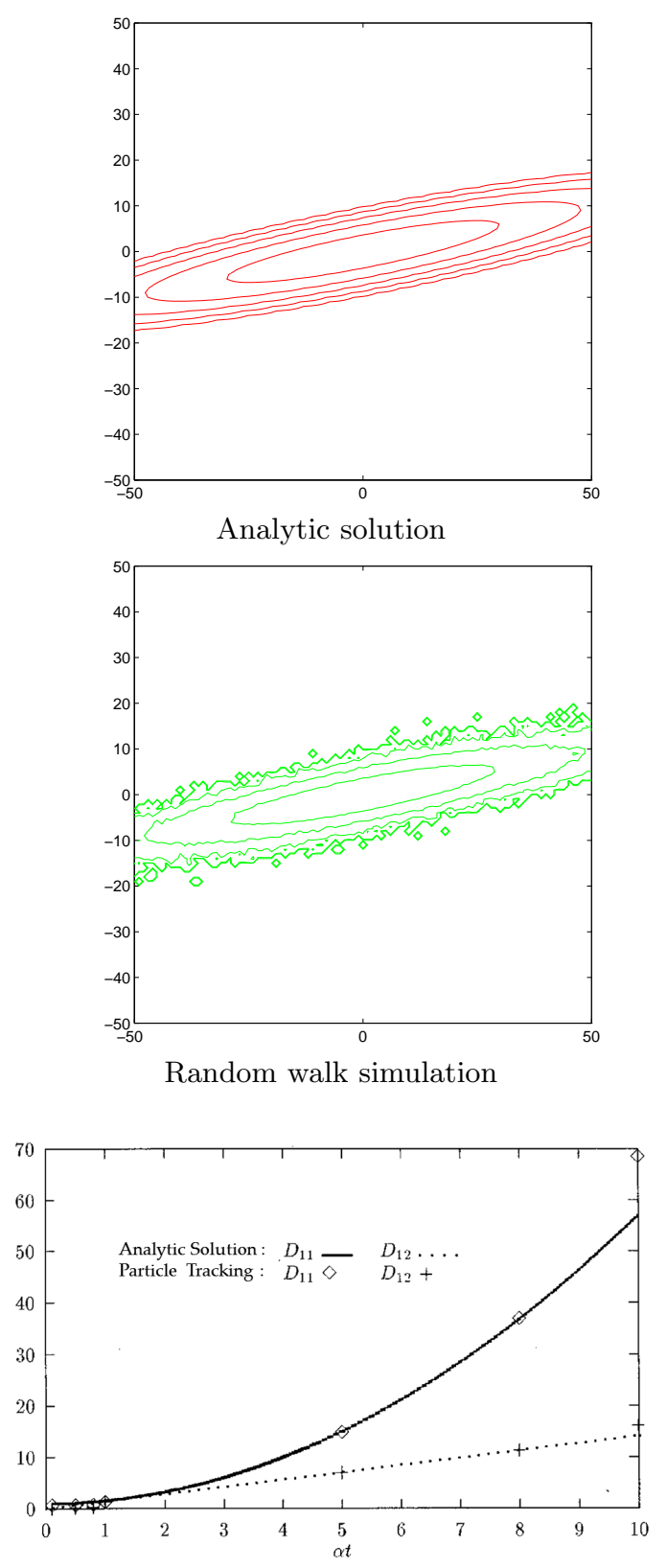

Diffusion coefficients $D_{i j}$ as a function of time $t, \alpha=$ shear rate

Figure 1. Dispersion of an instantaneous point source of particles in a simple shear flow. Comparison of the analytic solution of the kinetic equation for the particle spatial concentration and a random walk simulation based on Stokes drag with a Gaussian process for the aerodynamic driving force. For more precise details see [4, 7, 23]

has realizable solutions that are forward rather than backward in time consistent with a Gaussian process. We shall show that this is intimately related to the non Markovian nature of the kinetic equation, that the time evolution of the phase dispersion tensor from its initial state and the coupling between phase space variables are crucial considerations. In the course of 
this analysis we will recall the stages of the development of the kinetic equation and the important role played by certain consistency and invariance principles which taken together with the other features determining well-posedness and realizability have not been properly understood or appreciated in previous analyses.

Previous work has purported to show that the GLM is a more general approach than the kinetic approach. That in particular the kinetic equation can be derived from the GLM, and that the features of transport and mixing in more general non uniform inhomogeneous turbulent flows implicit in the solutions of the kinetic equation are intrinsic to the GLM. So the second objective of this analysis is to examine the basis for this assertion. In the process, we provide a more balanced appraisal of the benefits of both PDF approaches and point out the limitations of the GLM that have been ignored in previous analyses. We regard these limitations to be areas for improvement of the GLM rather than inherent deficiencies. Like all modeling approaches, each of the two approaches considered have their strengths and weaknesses. A categorical dismissal of one in preference to another in previous work would seem misplaced. From a practical point of view this paper is more about how one approach can support the other in solving dispersed flow problems.

\section{ILL-POSED KINETIC PDF EQUATIONS?}

In this section we examine in detail the previous analysis of Minier \& Profeta (M\&P) 12 that leads to the assertion of ill-posedness of the kinetic equation. For ease of comparison we use the same notation here and throughout the paper. Thus M\&P consider particle phase-space trajectories $\boldsymbol{Z}_{p}(t)=\left(\boldsymbol{X}_{p}(t), \boldsymbol{U}_{p}(t)\right)$ governed by

$$
\dot{\boldsymbol{X}}_{p}=\boldsymbol{U}_{p}, \quad \dot{\boldsymbol{U}}_{p}=\frac{1}{\tau_{p}}\left(\boldsymbol{U}_{s}-\boldsymbol{U}_{p}\right)+\boldsymbol{F}_{\text {ext }} .
$$

$\boldsymbol{U}_{s}(t)$ representing a flow velocity at time $t$ sampled along the trajectory $\boldsymbol{X}_{p}(t)$, and $\boldsymbol{F}_{\text {ext }}$ an external body force e.g. gravity. In the kinetic modeling framework, $\boldsymbol{U}_{s}$ is derived via an underlying flow velocity field $\boldsymbol{u}_{f}(\boldsymbol{x}, t)$ which has both a mean $\left\langle\boldsymbol{u}_{f}\right\rangle$ and fluctuating (zero mean component) $\boldsymbol{u}_{f}^{\prime}$. That is $\boldsymbol{U}_{s}=\boldsymbol{u}_{f}\left(\boldsymbol{X}_{p}(t), t\right)$. Treating $\boldsymbol{u}_{f}(\boldsymbol{x}, t)$ as a Gaussian stochastic flow field, and with the particle response time $\tau_{p}$ as a constant independent of the particle Reynolds no (i.e Stokes relaxation), the PDF $p(\boldsymbol{z}, t)$ defining the distribution of $\boldsymbol{Z}_{p}(t)$ then satisfies a transport equation (the kinetic equation) which can be written compactly in phase-space notation as_

$$
\partial_{t} p=-\partial_{\boldsymbol{z}} \cdot \boldsymbol{a} p+\frac{1}{2} \partial_{\boldsymbol{z}} \cdot\left(\partial_{\boldsymbol{z}} \cdot \boldsymbol{B} p\right)
$$

where $\boldsymbol{z}=(\boldsymbol{x}, \boldsymbol{v})$ refers to the particle position and velocity (in a fixed frame of reference) and

$$
\boldsymbol{a}=\left(\boldsymbol{v}, \boldsymbol{F}_{\text {ext }}+\frac{1}{\tau_{p}}\left(\left\langle\boldsymbol{u}_{f}(\boldsymbol{x}, t)\right\rangle-\boldsymbol{v}\right)+\boldsymbol{\kappa}\right)
$$

$$
\boldsymbol{B}=\left(\begin{array}{c|c}
\mathbf{0} & \boldsymbol{\lambda} \\
\hline \boldsymbol{\lambda}^{\top} & \boldsymbol{\mu}+\boldsymbol{\mu}^{\top}
\end{array}\right)
$$

$\boldsymbol{\lambda}$ and $\boldsymbol{\mu}$ are diffusion tensors that define gradient dispersion separately in real space $(\boldsymbol{x})$ and velocity space $(\boldsymbol{v})$ respectively. They are functions of time and depend on the particle response to the carrier flow velocity fluctuations along its trajectory. The specific forms for $\boldsymbol{\lambda}$ and $\boldsymbol{\mu}$ based on the LHDI closure scheme [18]

$$
\begin{aligned}
& \boldsymbol{\lambda}=\tau_{p}^{-2} \int_{0}^{t} \boldsymbol{g}^{T}(t-s) \cdot\left\langle\boldsymbol{u}_{f}^{\prime}(\boldsymbol{x}, \boldsymbol{v}, t \mid s) \boldsymbol{u}_{f}^{\prime}(\boldsymbol{x}, t)\right\rangle d s \\
& \boldsymbol{\mu}=\tau_{p}^{-2} \int_{0}^{t} \boldsymbol{g}^{T}(t-s) \cdot\left\langle\boldsymbol{u}_{f}^{\prime}(\boldsymbol{x}, \boldsymbol{v}, t \mid s) \boldsymbol{u}_{f}^{\prime}(\boldsymbol{x}, t)\right\rangle d s \\
& \boldsymbol{\kappa}=-\tau_{p}^{-2} \int_{0}^{t} \boldsymbol{g}^{T}(t-s) \cdot\left\langle\boldsymbol{u}_{f}^{\prime}(\boldsymbol{x}, \boldsymbol{v}, t \mid s) \partial_{\boldsymbol{x}} \boldsymbol{u}_{f}^{\prime}(\boldsymbol{x}, t)\right\rangle d s
\end{aligned}
$$

where the particle response tensor $\boldsymbol{g}(t-s)$ has elements $g_{i j}(t \mid s)$ corresponding to the displacement at time $t$ in the $j$-direction when $\tau_{p} u_{f}^{\prime}$ is an impulsive force $\delta(t-s)$ applied in the $i$-direction. In general $\boldsymbol{g}(t-s)$ depends up the local straining and rotation of the flow. Note the response tensor based on the Furutsu-Novikov closure scheme [26] is slightly different in definition (see Bragg \& Swailes [1] for a discussion of the different closure schemes for the kinetic equation). Following the analysis of $\mathrm{M} \& \mathrm{P}$, we consider the case for dispersion of an instantaneous point source in statistically stationary homogeneous and isotropic turbulence with a zero external force $\boldsymbol{F}_{\text {ext }}=0$ , in which case $\boldsymbol{g}(t)=\left(1-e^{-t / \tau_{p}}\right) \boldsymbol{I}$ and

$$
\begin{aligned}
& \boldsymbol{\lambda}=\tau_{p}^{-2} \int\left(1-e^{-s / \tau_{p}}\right) R(s) d s \boldsymbol{I} \\
& \boldsymbol{\mu}=\tau_{p}^{-2} \int e^{-s / \tau_{p}} R(s) d s \boldsymbol{I} \\
& \boldsymbol{\kappa}=0
\end{aligned}
$$

where $R(s)$ is the autocorrelation $\frac{1}{3}\left\langle\boldsymbol{U}_{s}^{\prime}(0) \cdot \boldsymbol{U}_{s}^{\prime}(s)\right\rangle$ of the flow velocity fluctuations $\boldsymbol{U}^{\prime}(s)$ measured along a particle trajectory. (2), (3)), (4) correspond to equations (65), (66), (67) in [12. M\&P claim that equation (2) is ill-posed in the sense that solutions to this can (will) exhibit unphysical behaviour except in special or, to use their phrase, 'lucky' cases. Specifically, they assert that solutions $p$ of equation (2) will exhibit finite-time singularities except for very special initial conditions, for example with a Gaussian form. Their justification for this claim is based on an analysis centered round the observation that $\boldsymbol{B}$ is not positive-definite but possesses both negative and positive eigenvalues. We show here that their analysis is incorrect.

Firstly we note that equation (2) is not a model for the PDF of $\boldsymbol{Z}_{p}(t)$, but describes precisely how this PDF must evolve. There is an exact correspondence between equation (2) and the underlying equation of motion (1). This equivalence, i.e. the formal derivation of (2) from (1), is subject only to the requirement that the field $\boldsymbol{u}_{f}(\boldsymbol{x}, t)$ is 
Gaussian. Then, notwithstanding the non-definiteness of $\boldsymbol{B}$, equation (2) is an exact description of how $p$, as determined by (1), behaves. Contrary to previous claims 12 no Gaussian (or other) constraint is necessary on the initial distribution $p^{0}(\boldsymbol{z})$ of $\boldsymbol{Z}_{p}(0)$. Thus, should solutions to (2) exhibit finite-time, or even asymptotic $(t \rightarrow \infty)$, singularities when $p^{0}$ is non-Gaussian, then this feature must be inherent in the system determined by (1). Either this singular behaviour is intrinsic to the system, or the analysis upon which M\&P base their conclusion is incorrect.

To demonstrate that the non-definiteness of $\boldsymbol{B}$, coupled with arbitrary initial conditions, does not lead to singular solutions of equation (2) we note that the solution to this equation can be written

$$
p(t ; \boldsymbol{z})=\int \phi\left(t ; \boldsymbol{z}, \boldsymbol{z}^{\prime}\right) p^{0}\left(\boldsymbol{z}^{\prime}\right) d \boldsymbol{z}^{\prime}
$$

where $\phi\left(t ; \boldsymbol{z}, \boldsymbol{z}^{\prime}\right)$ is the fundamental solution satisfying $\phi\left(0 ; \boldsymbol{z}, \boldsymbol{z}^{\prime}\right)=\delta\left(\boldsymbol{z}-\boldsymbol{z}^{\prime}\right)$. Now consider the case when $\boldsymbol{U}_{s}^{\prime}(t)=\boldsymbol{u}_{\boldsymbol{f}}{ }^{\prime}\left(\boldsymbol{X}_{p}, t\right)=\boldsymbol{u}_{\boldsymbol{f}}\left(\boldsymbol{X}_{p}, t\right)-\left\langle\boldsymbol{u}_{\boldsymbol{f}}\right\rangle\left(\boldsymbol{X}_{p}, t\right)$ is treated, ab initio, as a Gaussian process. The structure of equation (2) remains unchanged, except $\boldsymbol{\kappa} \equiv \mathbf{0}$ and $\boldsymbol{\lambda}, \boldsymbol{\mu}$ are independent of $\boldsymbol{Z}$ (but, crucially, they will still depend on $t$ ). $\boldsymbol{B}$ still has negative eigenvalues. With $\left\langle\boldsymbol{u}_{f}\right\rangle$ linear in $\boldsymbol{x}$ (and $\boldsymbol{F}_{\text {ext }}$ constant) the form of $\phi$ is well-documented, both in general terms and for a number of specific linear flows [4, 7, 20. This solution is Gaussian, and it is straightforward to show that it corresponds exactly, as it must, to the Gaussian form of $\boldsymbol{Z}_{p}$ determined by equation (1). Thus, any singular behaviour of the general solution $p$, defined by equation (5), can only be a consequence of degeneracy in the Gaussian form of $\phi$, and not the form of an arbitrary initial distribution $p^{0}$. Again, should such degeneracy exist then it would be symptomatic of behaviour determined by (1), and not some artifact of the non-definiteness of $\boldsymbol{B}$.

There are several flaws in the analysis upon which M\&P base their claim of ill-posedness: To begin, they consider a form of equation (2) in which $\boldsymbol{B}$ is taken as independent of time, arguing that this corresponds to stationary isotropic turbulence. This is not correct. $\boldsymbol{B}$ is intrinsically time dependent. This dependence reflects the non-zero time correlations implicit in the turbulent velocity field $\boldsymbol{U}_{f}$, and the consequent non-Markovian nature of $\boldsymbol{Z}_{p}$. Moreover, and crucially, $\boldsymbol{B}(0)=\mathbf{0}$ unless the initial values $\boldsymbol{U}_{p}(0), \boldsymbol{U}_{s}(0)$ are correlated. A detailed analysis of this is given in [7]. So, even when $\boldsymbol{B} \rightarrow \boldsymbol{B}^{\infty}$ (constant) as $t \rightarrow \infty$, it is inappropriate to set $\boldsymbol{B}=\boldsymbol{B}^{\infty}$ in a formal analysis of the time problem. Indeed, it is straightforward to show that the fundamental solution $\phi$ breaks down for arbitrarily small $t$ when this inappropriate approximation is introduced.

Of course, the non-definiteness of $\boldsymbol{B}$ is not altered by taking this tensor to be $t$ dependent. The eigensolution based transformation that M\&P introduce can still be invoked. Analogous to equation (71) in [12] we define trajectories $\widetilde{\boldsymbol{Z}}_{p}(t)$ with components $\left(\widetilde{Z}_{p 1}, \widetilde{Z}_{p 2}\right)$ in a transformed phase space $\widetilde{\boldsymbol{z}}=\left(\widetilde{z}_{1}, \widetilde{z}_{2}\right)$ with

$$
\widetilde{\boldsymbol{Z}}_{p}(t)=\mathrm{P}^{\top} \cdot \boldsymbol{Z}_{p}(t)
$$

where $\mathrm{P}(t)$ is the transformation matrix determined by the (now time dependent) normalized eigenvectors of $\boldsymbol{B}$. Thus, $\mathrm{P}^{\top} \cdot \mathrm{P}=\mathrm{I}$ and $\mathrm{P}^{\top} \cdot \boldsymbol{B} \cdot \mathrm{P}=\boldsymbol{\Lambda}=\operatorname{diag}\left(\omega_{i}\right)$, with $\omega_{i}$ the eigenvalues of $\boldsymbol{B}$. We note that, in applying this to the $2 \mathrm{D}$ case considered by the $\mathrm{M} \& \mathrm{P}$, it is sensible to label the two eigenvalues such that $\omega_{1}<0, \omega_{2}>0$ since this gives $\mathrm{P}(0)=\mathrm{I}$. By neglecting the time dependence in $\boldsymbol{B}$ $\mathrm{M} \& \mathrm{P}$ missed this point and chose the opposite ordering (see equation (69) in [12]). Here we take $\omega_{1}<0$.

In using the transform given by equation (6) it is important to note that equation (1) governing $\boldsymbol{Z}_{p}(t)$ is not to be interpreted as a stochastic differential equation driven by a white-noise process, and equation (2) is not a corresponding Fokker-Planck equation. Clearly this would be nonsense since $\boldsymbol{B}$ is not positive-definite. It is more transparent (and correct) to note that equation (6) implies that the PDF $\widetilde{p}(\widetilde{\boldsymbol{z}}, t)$ of $\widetilde{\boldsymbol{Z}}_{p}(t)$ is related to the PDF $p(\boldsymbol{z}, t)$ of $\boldsymbol{Z}_{p}(t)$ by $\widetilde{p}|\mathrm{~J}|=p$, where $\mathrm{J}=\operatorname{det}[\mathrm{P}]$ is the Jacobean of the transform $\widetilde{z}=\mathrm{P}^{\top} \cdot \boldsymbol{z}$. Since $\mathrm{P}$ is orthogonal we have $\mathrm{J}=1$. The PDF equation for $\widetilde{p}$ is

$$
\partial_{t} \widetilde{p}=-\partial_{\tilde{\boldsymbol{z}}} \cdot \widehat{\boldsymbol{a}} \widetilde{p}+\frac{1}{2} \partial_{\widetilde{\boldsymbol{z}}} \cdot\left(\partial_{\widetilde{\boldsymbol{z}}} \cdot \boldsymbol{\Lambda} \widetilde{p}\right)
$$

where $\widehat{\boldsymbol{a}}=\mathrm{P}^{\top} \cdot \widetilde{\boldsymbol{a}}+\mathrm{R} \cdot \widetilde{\boldsymbol{z}}, \widetilde{\boldsymbol{a}}(\widetilde{\boldsymbol{z}}, t)=\boldsymbol{a}(\boldsymbol{z}, t), \mathrm{R}=\dot{\mathrm{P}}^{\top} \cdot \mathrm{P}$. This is analogous to equation (72) in [12], except these authors have not included the time dependence in $\boldsymbol{B}$ and so set $\dot{P}=0$. We note that $R$ represents a rate of rotation matrix, $\operatorname{trace}(\mathrm{R})=0$. In the $2 \mathrm{D}$ model considered, the authors integrate equation (7) over $\widetilde{z}_{2}$ (corresponding to the transformed variable with the positive eigenvalue $\omega_{2}$ ) to obtain (compare with equation (74) in [12])

$$
\partial_{t} \widetilde{p}_{r}=-\partial_{\widetilde{\mathbf{z}}_{1}} \overline{\widehat{a}}_{1} \widetilde{p}_{r}-\partial_{\overline{\mathbf{z}}_{1}}^{2} \frac{1}{2}\left|\omega_{1}\right| \widetilde{p}_{r}
$$

where $\widetilde{p}_{r}$ is the PDF for $\widetilde{Z}_{p 1}$ and $\overline{\mathrm{a}}_{1} \widetilde{p}_{r}=\int \widehat{\mathrm{a}}_{1} \widetilde{p} d \widetilde{\mathrm{z}}_{2}$. Based on the negative diffusion coefficient in equation (8) $\mathrm{M} \& \mathrm{P}$ seek to show that this equation and so also equation (2) is ill-posed. Their argument fails to take into account that the conditional average $\overline{\widehat{a}}_{1}$ is a density weighted average, i.e its value at $z_{1}$ is dependent upon the distribution of $Z_{p 2}(t)$ at $z_{1}$ which itself can be a function $z_{1}$. For instance using a more explicit notation we may write

$$
\overline{\mathrm{a}}_{1} \equiv\left\langle\widehat{\mathrm{a}}_{1}\left(\widetilde{\mathrm{z}}_{1}, \widetilde{\mathrm{Z}}_{p 2}(t)\right)\right\rangle_{\widetilde{\mathrm{z}}_{1}}
$$

where $\langle\cdot\rangle_{\widetilde{z}_{1}}$ denotes an ensemble average conditioned on $\widetilde{\mathrm{Z}}_{p 1}(t)=\widetilde{\mathrm{Z}}_{1}$. What equation (9) illustrates is that only a sub-set of all trajectories $\widetilde{Z}_{p 2}(t)$ contribute to $\overline{\widehat{a}}_{1}$, namely those that are also associated with $\widetilde{\mathrm{Z}}_{p 1}(t)=\widetilde{\mathrm{Z}}_{1}$. The term $\overline{\widehat{a}}_{1}$ is therefore affected by coupling between $\widetilde{Z}_{p 1}(t)$ and $\widetilde{\mathrm{Z}}_{p 2}(t)$. Indeed, in the case where $\widetilde{\mathrm{Z}}_{p 1}(t)$ and $\widetilde{\mathrm{Z}}_{p 2}(t)$ are statistically decoupled, we have

$$
\left\langle\widehat{\mathrm{a}}_{1}\left(\widetilde{\mathrm{z}}_{1}, \widetilde{\mathrm{Z}}_{p 2}(t)\right)\right\rangle_{\widetilde{\mathrm{z}}_{1}}=\left\langle\widehat{\mathrm{a}}_{1}\left(\widetilde{\mathrm{z}}_{1}, \widetilde{\mathrm{Z}}_{p 2}(t)\right)\right\rangle,
$$


i.e. all realizations of $\widetilde{\mathrm{Z}}_{p 2}(t)$ would contribute to $\overline{\mathrm{a}}_{1}$. In this case $\overline{\mathrm{a}}_{1}\left(z_{1}\right)$ is convective as M\&P have assumed. However, in general, $\widetilde{\mathrm{Z}}_{p 1}(t)$ and $\widetilde{\mathrm{Z}}_{p 2}(t)$ will be statistically coupled, and as a consequence $\overline{\widehat{a}}_{1}$ cannot be treated as an arbitrary convective term. Indeed as we shall show momentarily, the term $\overline{\widehat{a}}_{1}$ is associated with both convective and diffusive fluxes, and its diffusional contribution offsets that associated with the negative eigenvalue.

By failing to appreciate this particular property of $\overline{\mathrm{a}}_{1}$, M\&P [12] have overlooked a fundamental property of the particle dispersion process. That is in the dynamical system described by equation (1), the particle position and velocity are not independent. This is reflected in the fixed-frame kinetic equation (2) through the term $\partial_{x} v p$, which couples the spatial and velocity distributions of the particles. In the same way, the distributions of the variables $\widetilde{Z}_{p 1}, \widetilde{Z}_{p 2}$ are coupled in equation (8). The implication of this coupling is that fluctuations in particle velocity give rise to fluctuations in particle position, in addition to the fluctuations in particle position that arise directly from fluctuations in the fluid force $\tau_{p}^{-1} \boldsymbol{U}_{s}$. In the moving frame it is the fluctuations in $\widetilde{Z}_{p 2}$ (with the +ve eigenvalue, $\omega_{2}$ ) via the $+v e$ covariance between between $\widetilde{Z}_{p 1}$ and $\widetilde{Z}_{p 2}$, that overcomes the -ve diffusion associated with $\widetilde{Z}_{p 1}$ (in the absence of the coupling). We note, for instance, that in equation (2), the particle flux $\boldsymbol{v} p$ integrated over all particle velocities is expressible as a net gradient diffusion flux,$\overline{\boldsymbol{v}} p_{r}$ for which the long term $(t \rightarrow \infty)$ particle diffusion coefficient $\varepsilon(\infty)$ in statistically stationary, homogeneous, isotropic turbulence is given by

$$
\varepsilon(\infty)=\tau_{p}\left\{\left\langle v^{2}(\infty)\right\rangle+\lambda(\infty)\right\}
$$

where $\left\langle v^{2}(\infty)\right\rangle$ is the variance of the particle velocity (which for a Gaussian process is given by $\left(\tau_{p} / 3\right) \operatorname{trace}(\boldsymbol{\mu}(\infty))$, see e.g equations (78-79) in [18]), and $\lambda=(1 / 3) \operatorname{trace}(\boldsymbol{\lambda})$. This simple relationship clearly identifies the two sources of dispersion independently, the first from fluctuations in the particle velocity (the kinetic contribution) and the second term $\lambda(\infty)$ arising from fluctuations in $\tau_{p}^{-1} \boldsymbol{U}_{s}$ (the turbulent aerodynamic force contribution). We refer to [17] for a detailed analysis of how this relationship defines an equation of state for the particle pressure and where $\left\langle v^{2}(\infty)\right\rangle$ and $\lambda(\infty)$ are more correctly identified as the normal components of stress tensors. We refer to [16] on how a proper treatment of the integrated flux terms in the kinetic equation in inhomogeneous turbulence gives rise to turbophoresis, an important mechanism for particle deposition (in response the unfounded criticism in both [11, 12] that the kinetic equation is inappropriate for modeling particle deposition).

To demonstrate these features in a quantitative way we consider the simple $2 \mathrm{D}$ case examined by $\mathrm{M} \& \mathrm{P}$ in which $\langle\boldsymbol{U}\rangle=\mathbf{0}$, and $\widetilde{\boldsymbol{Z}}_{p}(0)=\widetilde{\boldsymbol{z}}^{0}$ fixed. Then $\widehat{\mathbf{a}}$ is linear in $\widetilde{\boldsymbol{z}}$, and $\overline{\mathrm{a}}_{1} \widetilde{p}_{r}$ involves $\overline{\widetilde{\mathrm{z}}}_{2} \widetilde{p}_{r}=\int \widetilde{\mathrm{z}}_{2} \widetilde{p} d \widetilde{\mathrm{z}}_{2}$. This can be expressed in terms of convective and gradient diffusive fluxes (see [26])

$$
\overline{\widetilde{\mathrm{Z}}}_{2} \widetilde{p}_{r}=\widetilde{m}_{2} \widetilde{p}_{r}-\widetilde{\theta}_{21} \partial_{\widetilde{\mathbf{z}}_{1}} \widetilde{p}_{r}
$$

where $\widetilde{m}_{2}, \widetilde{\theta}_{21}$ are components of $\left\langle\widetilde{\boldsymbol{Z}}_{p}\right\rangle=\widetilde{\boldsymbol{m}}=\left(\widetilde{m}_{1}, \widetilde{m}_{2}\right)$ and $\left\langle\left(\widetilde{\boldsymbol{Z}}_{p}-\widetilde{\boldsymbol{m}}\right)\left(\widetilde{\boldsymbol{Z}}_{p}-\widetilde{\boldsymbol{m}}\right)\right\rangle=\widetilde{\Theta}=\left(\widetilde{\theta}_{i j}\right)$ satisfying

$$
\begin{aligned}
\dot{\boldsymbol{m}} & =\tilde{\boldsymbol{\Gamma}} \cdot \widetilde{\boldsymbol{m}}+\widetilde{\boldsymbol{k}} \\
\dot{\widetilde{\Theta}} & =\tilde{\boldsymbol{\Gamma}} \cdot \widetilde{\Theta}+(\tilde{\boldsymbol{\Gamma}} \cdot \widetilde{\Theta})^{\mathrm{T}}+\Lambda
\end{aligned}
$$

with $\widetilde{\boldsymbol{m}}(0)=\widetilde{\boldsymbol{z}}^{0}, \widetilde{\Theta}(0)=\mathbf{0}$. Here $\tilde{\boldsymbol{\Gamma}}=\mathrm{P}^{\mathrm{T}} \cdot \mathbf{A} \cdot \mathrm{P}+\mathrm{R}, \widetilde{\boldsymbol{k}}=$ $\mathrm{P}^{\mathrm{T}} \cdot \boldsymbol{k}$ with $\boldsymbol{k}=\left(\mathbf{0}, \boldsymbol{F}_{\text {ext }}\right)$ and $\mathrm{A}_{11}=\mathrm{A}_{21}=0, \mathrm{~A}_{12}=1$, $\mathrm{A}_{22}=-1 / \tau_{p}^{\mathrm{St}}$. equations (12), 13), 14) allow equation (8) to be written

$$
\partial_{t} \widetilde{p}_{r}=-\partial_{\overline{\mathrm{z}}_{1}} \dot{\tilde{m}}_{1} \widetilde{p}_{r}+\partial_{\mathrm{z}_{1}}^{2} \frac{1}{2} \dot{\widetilde{\theta}}_{11} \widetilde{p}_{r}
$$

The net diffusional effect is therefore determined by the particle diffusion coefficient $\widetilde{D}_{1}(t)$ of the transformed variable $\tilde{z}_{1}$ (associated with the negative eigenvalue $\omega_{1}$ ) and given by

$$
\widetilde{D}_{1}(t)=\frac{1}{2} \dot{\widetilde{\theta}}_{11}=(\tilde{\boldsymbol{\Gamma}} \cdot \widetilde{\Theta})_{11}-\frac{1}{2}\left|\omega_{1}\right|
$$

This shows how the 'anti-diffusion' associated with $\omega_{1}$ is offset by the contribution emerging from the flux $\overline{\mathrm{a}}_{1} \widetilde{p}_{r}$ associated with the coupling between $\widetilde{Z}_{p 1}$ and $\widetilde{Z}_{p 2}$ through their covariance $\widetilde{\theta}_{12}$ in equation 16 .

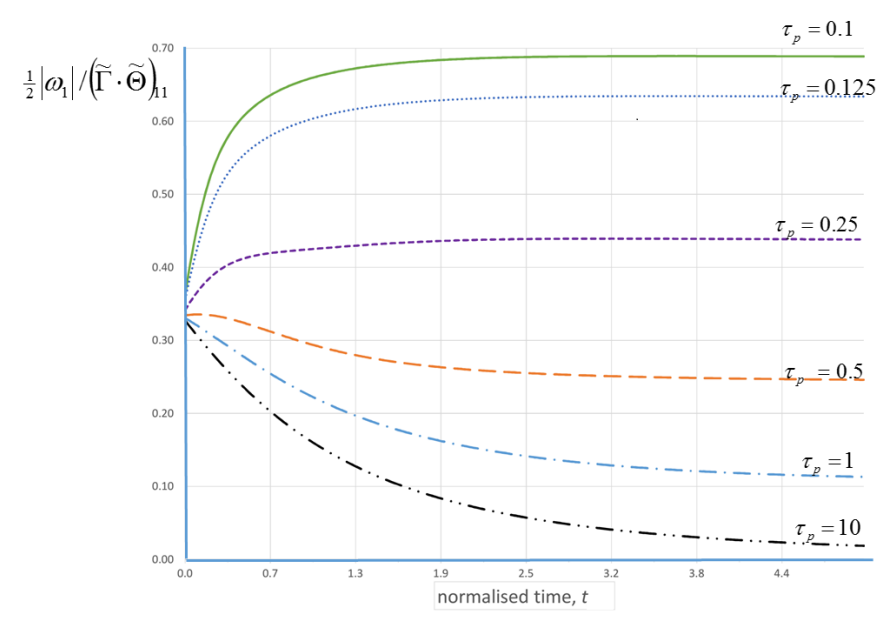

Figure 2. Plots of $\frac{1}{2}\left|\omega_{1}\right| /\left(\tilde{\boldsymbol{\Gamma}} \cdot \widetilde{\Theta}_{11}\right)$ equation 16 for the ratio of $-v e /+v e$ contributions to the particle diffusion coefficient $\widetilde{D}_{1}$ of the transformed variable $\widetilde{Z}_{p 1}$ (with a $-v e$ eigenvalue) in the moving frame of reference, as a function of time $t$ for a range of values of the particle response time $\tau_{p}$. Both $t$ and $\tau_{p}$ are scaled on $T_{L}$, the Lagrangian integral timescale of the carrier flow measured along a particle trajectory.

Figure 2 demonstrates that $0 \leqslant \frac{1}{2}\left|\omega_{1}\right| /(\tilde{\boldsymbol{\Gamma}} \cdot \widetilde{\Theta})_{11} \leqslant 1$. The plots, which show the time evolution of this ratio 
for a range of values for $\tau_{p}$ (with $\mathbf{F}_{\text {ext }}=\mathbf{0}$ ), were obtained from closed form solutions of (14). These solutions are constructed by noting that $\widetilde{\boldsymbol{\Theta}}=\mathrm{P}^{\mathrm{T}} \cdot \boldsymbol{\Theta} \cdot \mathrm{P}$, where the covariances $\mathbf{\Theta}=\left\langle\mathbf{Z}_{p} \mathbf{Z}_{p}\right\rangle$ in the fixed frame are governed by a set of equations analogous to equations (14) 17 which can be integrated analytically. We refer to 17. where analytic solutions are given for $\boldsymbol{\Theta}$ in terms of $\left\langle\mathrm{U}_{s}^{\prime}(0) \mathrm{U}_{s}^{\prime}(t)\right\rangle$ the autocorrelation of the carrier flow velocity fluctuations sampled along particle trajectories. The values of the $-v e$ to $+v e$ ratio plotted in Figure 2 were obtained using an exponential decay $\exp \left[-t / T_{L}\right]$ for this autocorrelation. For completeness we also show in Figure 3 for a similar range of values of $\tau_{p}$, the evolution of the particle diffusion coefficient $\widetilde{D}_{1}(t)$ in the moving frame of reference indicating not only that $\widetilde{D}_{1} \geqslant 0$, but also that it reaches an asymptotic limit that is the same for all $\tau_{p}$. This is is also true of the particle diffusion coefficient $\varepsilon(\infty)$ in the fixed frame of reference, equation (11). In particular in the normalised units used to express the values for $\widetilde{D}_{1}$ in Figure $3, \varepsilon(\infty)=1$. This result is universally true for a particle equation of motion involving the linear drag form in equation (1) for statistically stationary homogeneous isotropic turbulence (see 14 where it is $T_{L}$ that depends on $\tau_{p}$ ). An evaluation of the asymptotic form of $\left\langle\mathbf{Z}_{p} \mathbf{Z}_{p}\right\rangle$ which is linear in $t$ in this limit shows that

$$
\begin{aligned}
& \widetilde{D}_{1}(\infty)=1 /(4-2 \sqrt{2}) \\
& \widetilde{D}_{2}(\infty)=1 /(4+2 \sqrt{2})
\end{aligned}
$$

and is consistent with the forms for $\widetilde{D}_{1}(t)$ in Figure 3 obtained by solving a coupled set of equations (14) for $\widetilde{\boldsymbol{\Theta}}$. That the asymptotic result in equation 17 agrees with the results in Figure 3 provides not only a check for the analytic solutions used in Figure 3 , but also a proof that the +ve contribution to $\widetilde{D}_{1}(t)$ will always outweigh the $-v e$ contribution in equation (16) (i.e. it applies to all physically acceptable forms of the autocorrelation for $\boldsymbol{U}_{s}$, and not just the decaying exponential form of $\left\langle\mathrm{U}_{s}^{\prime}(0) \mathrm{U}_{s}^{\prime}(t)\right\rangle$ that we have chosen to obtain our analytical results).

This must be so for two reasons. Firstly the route involving a solution of the kinetic equation in the fixed frame of reference and the linear relationship between the fixed and transformed variables always ensures a realizable Gaussian distribution for the transformed variables. Secondly in this calculation this realizability does not itself explicitly involve or rely in any way on whether one of the eigenvalues $\omega_{i}<0$ and any explicit form for $\left\langle\mathrm{U}_{s}^{\prime}(0) \mathrm{U}_{s}^{\prime}(t)\right\rangle$ we might choose, only that the transformation matrix $\boldsymbol{P}$ formed from the normalised eigenvectors of the diffusion matrix exists and is well behaved. However the second route via equation (14) only ensures a realizable Gaussian process if the +ve contribution to $\widetilde{D}_{1}(t)$ exceeds the $-v e$ contribution. But since the two methods of calculating $\widetilde{\Theta}$ are in the end mathematically equivalent to one another, then the +ve contribution to $\widetilde{D}_{1}(t)$ must always exceed the $-v e$ contribution in equation (16).

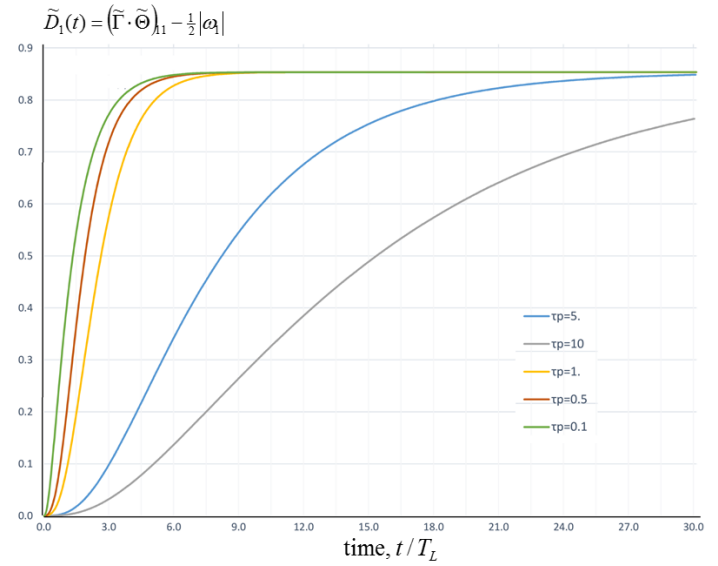

Figure 3. Evolution of the particle diffusion coefficient $\widetilde{D}_{1}(t)$ evaluated using equation 16 in the moving frame of reference for a range of values of $\tau_{p}$ (the particle response time normalised on the Lagrangian integral time scale, $\left.T_{L}\right)$. Time is real time $t$ normalised on $T_{L}$.

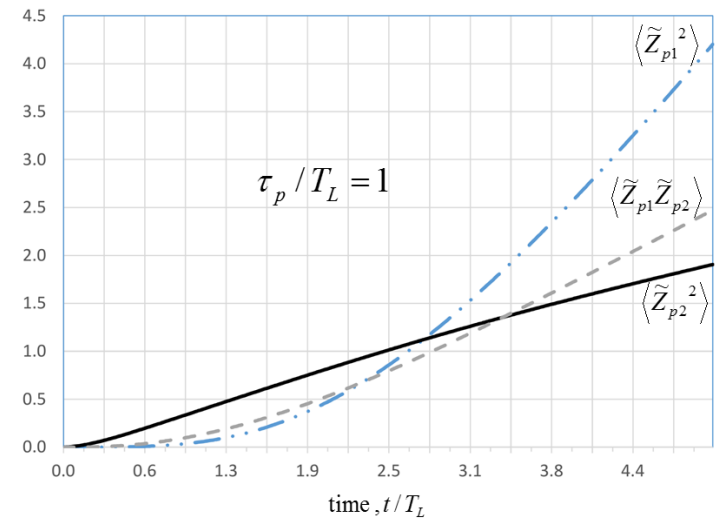

Figure 4. Moments $\left\langle\widetilde{Z}_{p i} \widetilde{Z}_{p j}\right\rangle$ in the moving frame of reference based on the moments $\left\langle\boldsymbol{Z}_{p} \boldsymbol{Z}_{p}\right\rangle$ for $\tau_{p} / T_{L}$ in the fixed frame of reference as solutions of the fixed frame kinetic equation (2) or equivalently by evaluating $\left\langle\boldsymbol{Z}_{p} \boldsymbol{Z}_{p}\right\rangle$ from solutions of the particle equation of motion equation (1).

We show the values of the moments $\left\langle\widetilde{Z}_{p i} \widetilde{Z}_{p j}\right\rangle$ in Figure 4 appropriate for the Gaussian function solution of the kinetic equation in the moving frame (see equation (87) in 17). There is of course no hint of a singularity in Figure 4, all 3 moments being smoothly varying, monotonically increasing in time and linear in time for $t / T \gg 1$.

The results also illustrate the now obvious result that, at large times, the two contributions to the diffusional transport are of the same order in $t$. The claim in 12 that equation (8) reduces to the form of a backward heat equation because $\overline{\mathrm{a}}_{1} \widetilde{p}_{r} \rightarrow 0$ as $t \rightarrow \infty$ is invalid. It fails to acknowledge that $\omega_{1} \partial_{\widetilde{\mathbf{z}}_{1}} \widetilde{p}_{r} \rightarrow 0$ at the same rate.

Although we have now demonstrated that the trans- 
formed kinetic equation is not ill-posed, we close this section with some comments on M\&P's use of the Feynman-Kac formula (FKF) and the associated arguments in 12. In 12, M\&P suggest that equation (8) has the structure of a (generalized) Backward Kolmogorov Equation (BKE), that may be derived from FKF. Noting this, M\&P use the FKF to construct the solution to equation (8), using the terminal condition $\widetilde{p_{r}}\left(\widetilde{\mathrm{z}}_{1}, T\right)=\Psi\left(\widetilde{\mathrm{z}}_{1}\right)$, to obtain $(t \in[0, T])$

$\widetilde{p_{r}}\left(\widetilde{\mathrm{z}}_{1}, t\right)=\left\langle\exp \left[\int_{t}^{T} \partial_{\widetilde{\mathbf{z}}_{1}} \overline{\mathrm{a}}_{1}(\mathcal{X}(s), s) d s\right] \Psi(\mathcal{X}(T))\right\rangle_{\mathcal{X}(t)=\widetilde{\mathbf{z}}_{1}}$,

where $\mathcal{X}(s)$ is a stochastic process defined through

$$
d \mathcal{X}(s) \equiv \overline{\widehat{a}}_{1}(\mathcal{X}(s), s) d s+\sqrt{\left|\omega_{1}(s)\right|} d W(s),
$$

and $W(s)$ is a Wiener process. M\&P argue that the solution (18) implies that only "special" initial $(t=0)$ conditions are permitted when solving (8) since (18) specifies

$\widetilde{p_{r}}\left(\widetilde{\mathrm{z}}_{1}, 0\right)=\left\langle\exp \left[\int_{0}^{T} \partial_{\widetilde{\mathbf{z}}_{1}} \overline{\widehat{\mathrm{a}}}_{1}(\mathcal{X}(s), s) d s\right] \Psi(\mathcal{X}(T))\right\rangle_{\mathcal{X}(0)=\widetilde{\mathbf{z}}_{1}}$

From this they conclude that since equation (18) only applies for the "special initial condition" given by equation (20), then equation (8) "is an unstable and ill-posed equation." This conclusion is clearly erroneous. Since the FKF employs a terminal condition in solving the PDE, then provided the PDE is well-posed as a terminal-value problem, the solution of the PDE at $t=0$ must of necessity be unique and "special". For a well-posed, deterministic PDE, there exists only one solution at $t=0$ that generates the specified terminal condition at $t=T$, otherwise solutions to the PDE are not unique!

If equation (8) were truly a BKE, then it could indeed be considered ill-posed since the BKE is in general ill-posed when solved as a time-forward problem (and equation (8) is to be solved as a time-forward problem with a prescribed initial condition). However, the important point is that although equation (8) superficially appears to have the structure of a BKE, it cannot be considered to be equivalent to a BKE for two reasons. First, as we have already discussed, the term $\overline{\mathrm{a}}_{1}$ is not a general convection term, but has a specific form since it is a functional of the solution of the equation (8). This is in part a manifestation of the fact that unlike the BKE, equation (8) is in fact derived from an underlying process that takes place in a higher dimensional space (i.e the phase-space). Second, equation (8) is associated with a non-Markovian process, whereas the BKE corresponds to a Markov process. The implication of this is that equation (18) cannot, at least formally, cover the entire solution space of the PDE in equation (8), since equation (8) admits solutions that correspond to non-Markov trajectories in the space $\widetilde{\mathrm{z}}_{1}$, which equation 18 does not account for since it constructs solutions via a conditional expectation over Markov trajectories. Therefore, in the general case, the FKF cannot be used to say anything categorical regarding the solutions to equation (8).

\section{KINETIC AND GLM EQUATIONS}

It has been claimed in recent studies of PDF methods [11, 12], that the kinetic PDF is the marginal of the GLM PDF. This claim is based on analysis that purports to show that the dispersion tensors appearing in a kinetic PDF equation derived from the GLM PDF equation are 'strictly identical' to the corresponding tensors emerging directly from the kinetic modeling approach. If this is so the claim of ill posedness of the kinetic equation contradicts the well posedness associated with the FokkerPlanck equation of the GLM. Of course, as we have just demonstrated, this claim of ill-posedness is ill founded. Here we consider the validity of the analysis presented in [12 to demonstrate how the kinetic equation can be derived from the GLM PDF equation.

The analysis is based on the construction of a closure for $\left\langle\boldsymbol{u}_{s} \mathcal{P}\right\rangle$ where $\boldsymbol{u}_{s}(t ; \boldsymbol{x})=\boldsymbol{U}_{s}(t)-\left\langle\boldsymbol{U}_{s}(t) \mid\left(\boldsymbol{X}_{p}(t)=\boldsymbol{x}\right)\right\rangle$ and $\left.\mathcal{P}(\boldsymbol{x}, \boldsymbol{v}, t)=\delta\left(\boldsymbol{X}_{p}(t)-\boldsymbol{x}\right) \delta\left(\boldsymbol{U}_{p}(t)-\boldsymbol{v}\right)\right)=\delta\left(\boldsymbol{Z}_{p}(t)-\right.$ $\boldsymbol{z})$. We make the simple observation that the ensemble $\langle\cdot\rangle$ to be considered in this closure involves all realizations of the system being considered. It is not, nor can it be interpreted as an average over only those realizations in which the trajectories $\boldsymbol{Z}_{p}$ satisfy the end-condition $\boldsymbol{Z}_{p}(t)=\boldsymbol{z}$. Indeed this is why $\left\langle\boldsymbol{u}_{s} \mathcal{P}\right\rangle=\left\langle\boldsymbol{u}_{s}\right\rangle_{\mathbf{z}} p(\boldsymbol{z}, t)$, where $\langle\cdot\rangle_{\mathbf{z}}$ denotes an average based on the sub-ensemble containing only those trajectories satisfying this end-condition. Although self-evident, this point is missed in the closure formulated in [12. This closure is constructed by introducing paths $\boldsymbol{\omega}(s)=\boldsymbol{\omega}(s ; \boldsymbol{z}, t)$ such that $(\boldsymbol{\omega}(t), \dot{\boldsymbol{\omega}}(t))=\boldsymbol{z}$. These paths are used to partition particle trajectories; for a given path $\boldsymbol{\omega}(\cdot ; \boldsymbol{z}, t)$ define $\boldsymbol{\Omega}_{\boldsymbol{\omega}}=\left\{\boldsymbol{Z}_{p}: \boldsymbol{X}_{p}(s)=\right.$ $\boldsymbol{\omega}(s ; \boldsymbol{z}, t)\}$. In 12 a closure is then considered for the sub-ensemble $\left\langle\boldsymbol{u}_{s} \mathcal{P}\right\rangle^{\boldsymbol{\Omega}_{\boldsymbol{\omega}}}$ over those trajectories in $\boldsymbol{\Omega}_{\boldsymbol{\omega}}$ (see equation (39) in [12]), and this closure is then integrated over all paths $\boldsymbol{\omega}(\cdot ; \boldsymbol{z}, t)$. Thus, only trajectories satisfying the specified end-condition $\boldsymbol{Z}_{p}(t)=\boldsymbol{z}$ have been taken into account. This is wrong. Moreover, the form of the closure for $\left\langle\boldsymbol{u}_{s} \mathcal{P}\right\rangle^{\boldsymbol{\Omega}_{\boldsymbol{\omega}}}$ is questionable. The FurutsuNovikov formula is invoked, the correct application of this should result in a closure framed in terms of the two-time correlation tensor $\mathrm{C}\left(s, s^{\prime} ; \boldsymbol{z}, t\right)=\left\langle\boldsymbol{u}^{\boldsymbol{\omega}}(s) \boldsymbol{u}^{\boldsymbol{\omega}}\left(s^{\prime}\right)\right\rangle^{\boldsymbol{u}^{\boldsymbol{\omega}}}$ of the process $\boldsymbol{u}^{\boldsymbol{\omega}}(s)=\boldsymbol{u}_{s}(\boldsymbol{\omega}(s ; \boldsymbol{z}, t), s)$. However, in [12] this is conflated with another correlation, namely

$$
R\left(s, \boldsymbol{x} ; s^{\prime}, \boldsymbol{x}^{\prime}\right)=\left\langle\boldsymbol{u}_{s}(s ; \boldsymbol{x}, t) \boldsymbol{u}_{s}\left(s^{\prime} ; \boldsymbol{x}^{\prime}, t^{\prime}\right)\right\rangle
$$

Again, this is evidently wrong; $\mathrm{C}$ depends on a single phase-space point, $\boldsymbol{z}$, whereas $R$ is defined in terms of two points $\boldsymbol{x}, \boldsymbol{x}^{\prime}$ in configuration space. Not only this, the ensembles over which these two correlation tensors 
are constructed are different. Finally (and notwithstanding these apparent oversights), even if the resulting forms of the dispersion tensors emerging from the construction given in [12] were correct, it is incorrect to claim that these tensors are identical to those appearing in the PDF equation of the kinetic model. In the kinetic PDF equation the dispersion tensors are defined in terms of the basic two-point, two-time correlation tensor of the underlying fluctuations in the carrier flow velocity field, that is $\mathcal{R}\left(\boldsymbol{x}, t ; \boldsymbol{x}^{\prime}, t^{\prime}\right)=\left\langle\boldsymbol{u}^{\prime}(\boldsymbol{x}, t) \boldsymbol{u}^{\prime}\left(\boldsymbol{x}^{\prime}, t^{\prime}\right)\right\rangle$. This makes no reference to particle trajectories and, therefore, $\mathcal{R}$ cannot be deemed identical to $R$ defined by equation 21 .

\section{LIMITATIONS OF THE GLM FOR DISPERSED PARTICLE FLOWS}

That the GLM is a model and not a fundamental theory of particle dispersion in turbulent flows, is not an issue of critical concern. Like all models it has its advantages as well as its limitations. For instance an obvious advantage is that the GLM PDF includes the flow velocity sampled along a particle trajectory as an additional statistical variable as well as the particle velocity and position. So a solution of the PDF equation in principle contains more information about the dispersion process than the solution of the kinetic equation. Most noticeably Simonin and his co workers have used this PDF equation to formulate transport equations for the density weighted mean flow velocity $\overline{\boldsymbol{U}_{s}}$ and the particle-flow covariances and obtained remarkably good agreement with experimental measurement in numerous particle laden flows including jets and vertical channel flows 23, 25. Van Dijk \& Swailes 29 solved this GLM PDF equation numerically directly in the case of particle transport and deposition in a turbulent boundary layer showing the existence of singularities in the near wall particle concentration. Reeks 19 solved this PDF equation for particle dispersion in a simple shear and obtained valuable insights into the influence of the shear on the fluid velocity correlations as well as the dispersion in the streamwise direction which showed a component of contra-gradient diffusion [19].

Our aim here is to point out the limitations of the GLM for dispersed gas-particle flows that have been ignored in previous analyses especially in [12], to give a more balanced view of its strengths and weaknesses when compared to the kinetic approach. We regard these limitations to be areas for improvement of the model rather than inherent deficiencies. The advantage of models of this sort is that features inherent in more fundamental approaches like the kinetic approach can be included in an ad hoc manner.

Central to the formulation of the GLM PDF equations is the need to model $\dot{\boldsymbol{U}}_{s}$ (equations (23), (24), (25) in [12]). By definition

$$
\dot{\boldsymbol{U}}_{s}(t)=\left(\frac{D \boldsymbol{u}_{f}}{D t}-\left(\boldsymbol{u}_{f}-\boldsymbol{U}_{p}\right) \cdot \partial_{\mathbf{x}} \boldsymbol{u}_{f}\right)_{\boldsymbol{x}=\boldsymbol{X}_{p}(t)}
$$

with $D \boldsymbol{u}_{f} / D t$ denoting the fluid acceleration field, and $(\cdot)_{\boldsymbol{x}=\boldsymbol{X}_{p}(t)}$ denoting that the field variables inside the parenthesis are evaluated at the particle position. Equation 22 shows that the process $\dot{\boldsymbol{U}}_{s}(t)$ is fundamentally connected to the properties of the underlying flow fields, and as such is influenced by the spatio-temporal structure of those fields. This is particularly important since it is known, for example, that inertial particles interact with the topology of fluid velocity fields in particular ways, with a preference to accumulate in the strain dominated regions of the flow 10. Equation 22 captures the way in which the process $\dot{\boldsymbol{U}}_{s}(t)$ is affected by the properties of the underlying flow fields. However, in the GLM, $\dot{\boldsymbol{U}}_{s}(t)$ is modeled using a Langevin equation, and as such, the influence of the spatio-temporal structure of the underlying fields on $\dot{\boldsymbol{U}}_{s}(t)$ is lost. This means then that the GLM cannot properly capture the role of flow structure on inertial particle dynamics in turbulent flows, which is known to be very important for describing the spatial distributions of the particles. In contrast to this, the kinetic model does capture the role of the spatio-temporal structure of the flow on the particle motion. For example, the dispersion tensors $\boldsymbol{\lambda}$, $\boldsymbol{\mu}$ and $\boldsymbol{\kappa}$ capture these effects through their dependence on the two-point, two-time correlation tensor of the fluid velocity field.

A second, related issue, concerns the handling of the term $\left(\boldsymbol{u}_{f}-\boldsymbol{U}_{p}\right) \cdot \partial_{\boldsymbol{x}} \boldsymbol{u}_{f}$ in the GLM. The role of this term in 22 is that it captures how the particle inertia causes the timescale of $\boldsymbol{U}_{s}(t)$ to deviate from the Lagrangian timescale of the fluid velocity. For example, in the limit $\tau_{p} \rightarrow 0$, one should recover $\dot{\boldsymbol{U}}_{s}=\left(D \boldsymbol{u}_{f} / D t\right)_{\boldsymbol{x}=\boldsymbol{X}_{p}(t)}$, while in the limit $\tau_{p} \rightarrow \infty$ (without body forces), one should recover $\dot{\boldsymbol{U}}_{s}=\left(\partial_{t} \boldsymbol{u}_{f}\right)_{\boldsymbol{x}=\boldsymbol{X}_{p}(t)}$. In the former case, the timescale of $\boldsymbol{U}_{s}$ is the fluid Lagrangian timescale, whereas in the latter case the timescale of $\boldsymbol{U}_{s}$ is the fluid Eulerian timescale. With body forces e.g. gravity, the timescale of $\boldsymbol{U}_{s}$ for inertial particles would also be affected by the crossing trajectories effect [30.

Conventionally, the term $\left(\boldsymbol{u}_{f}-\boldsymbol{U}_{p}\right) \cdot \partial_{\boldsymbol{x}} \boldsymbol{u}_{f}$ is either neglected, such that the Langevin model relates to $\dot{\boldsymbol{U}}_{s}=$ $\left(D \boldsymbol{u}_{f} / D t\right)_{\boldsymbol{x}=\boldsymbol{X}_{p}(t)}$, or else its effect is modeled by making the timescale in the Langevin model a function of $\tau_{p}$. Both approaches are problematic: the first because it neglects the effect of inertia on the timescale which can be strong, the second because one then requires an additional model for the timescale of $\boldsymbol{U}_{s}$ as a function of $\tau_{p}$. In contrast, in the kinetic model, the role of inertia on $\boldsymbol{U}_{s}$ is formally accounted for, and is an intrinsic part of the model. In particular, it is captured through the dependence of $\boldsymbol{\mu}, \boldsymbol{\lambda}$ and $\boldsymbol{\kappa}$ on the correlation tensors of the 
fluid velocity field evaluated along the inertial particle trajectories.

Another implication of the GLM's use of a Langevin equation to describe $\boldsymbol{U}_{s}(t)$ is that, as is well known, it cannot accurately describe the Lagrangian properties of the system in the short-time 'ballistic' limit. For example, the second-order Lagrangian structure function $\left\langle\left\|\boldsymbol{U}_{s}(t+s)-\boldsymbol{U}_{s}(t)\right\|^{2}\right\rangle$ should grow as $s^{2}$ in the limit $s \rightarrow 0$, whereas a Langevin equation predicts that it grows linearly in $s$ in the limit $s \rightarrow 0$. Interestingly, this very fact has an important bearing on claim of the exact correspondence of the PDF of the kinetic equations with the marginal of the GLM PDF. Even aside from other issues, this claim cannot be correct since the kinetic model would give the correct short-time behavior for $\left\langle\left\|\boldsymbol{U}_{s}(t+s)-\boldsymbol{U}_{s}(t)\right\|^{2}\right\rangle$ since it allows for the general case where the fluid velocity field is differentiable in time.

In addition to these points, recent criticism of the kinetic equation failed to appreciate or show any awareness of important consistency and invariance principles that were important guidelines in the construction of the kinetic equation and highly relevant to the to the limitations and generality of the GLM PDF equations. The first is that the kinetic equation should generate the correct equation of state, i.e. the relation between the equilibrium pressure associated with the correlated turbulent motion of the particles and their mass density in homogeneous isotropic statistically stationary turbulence. This can be obtained independently of the kinetic equation by evaluating the Virial for the particle equation of motion (see section II in [17). This relates the kinematic pressure $\hat{p}$ to the particle diffusion coefficient $\varepsilon$ via the particle response time $\tau_{p}$, namely $\hat{p}=\varepsilon \tau_{p}^{-1}$.

The second important consideration is that the kinetic equation should satisfy Random Galilean Transformation (RGT) invariance [5, 8, 18, . In the development of legitimate closure schemes invariance to RGT is crucial to account for the transport of small scales of turbulence by the large scales and the $E(k) \sim k^{-5 / 3}$. Specifically RGT means applying to each realization of the carrier flow a translational velocity, constant in space and time but varying randomly in value from one realization to the next. In Kraichnan's traditional usage of RGT the distribution of velocities is taken to be Gaussian for convenience. Clearly the internal dynamics should be unaffected by this transformation and should be reflected in the equations that describe the average behavior of the resulting system. In the case of the case of the kinetic equation the terms that describe the dispersion due to the aerodynamic driving force and that due to the transnational velocity should be separate. When the timescale of $\boldsymbol{U}_{s}$ is finite, RGT cannot be satisfied by a PDF equation with the traditional Fokker-Planck structure. Indeed, RGT invariance implies that the dispersion tensor $\boldsymbol{B}$ in equation (2) must have the form given in equation (4) 17, which is not satisfied in a PDF equation with the Fokker-Planck structure (for which $\boldsymbol{\lambda} \equiv \mathbf{0}$ ).

This failure to preserve RGT invariance means fail- ure to reproduce the correct equation of state for the dispersed phase. In the case of the kinetic equation, it implies that that the dispersion tensor $\boldsymbol{B}$ in Eq. 2 must have the form given in Eq. (4) for a Gaussian non-white noise process. See 17] for the form of the dispersion tensor $\boldsymbol{B}$ for a non Gaussian process as a cumulant expansion in particle fluid velocity correlations. In the case of the GLM equations the failure to preserve RGT invariance is associated with the fluctuating stochastic acceleration field $\dot{\boldsymbol{U}}_{s}^{\prime}=\dot{\boldsymbol{U}}_{s}-\left\langle\dot{\boldsymbol{U}}_{s}\right\rangle$ described by a Fokker Planck equation. It is highlighted in the case of short term dispersion in e.g homogeneous station ary turbulence where the GLM predicts an exponential decay for the fluid velocity autocorrelation (along particle trajectories) and with it a discontinuity in the slope at $t=0$ and a consequent error in the short term dispersion of $\mathcal{O} t$ as opposed to $\mathcal{O} t^{2}$. Such a result cannot arbitrarily be changed since the exponential autocorrelation is a property of the white noise based GLM equation for all time.

This has some bearing on the equivalence of the two approaches, since the kinetic approach does not have this limitation and correctly predicts the short term diffusion. So whereas in the GLM, the form of the particle-flow correlations are calculated and an intrinsic part of the model, in the kinetic equation these are prescribed or calculated using independent knowledge of the statistics of the carrier flow field and a relationship between Eulerian and Lagrangian correlations. As pointed out in [19] in the case of dispersion in a simple shear flow, if the statistics of the fluid velocity along a particle trajectory are assumed derivable from a Gaussian process and the fluid velocity correlations as a function of time are taken to be the same in either case, then the two approaches are identical, but only then. Whilst in the kinetic equation one is free in principle to choose whatever is physically acceptable for the fluid particle correlation, the problem remains one of calculating carrier flow velocity correlations along particle trajectories, given the underlying Eulerian statistics of the carrier flow velocity field.

\section{The kinetic equation for non-linear drag}

In closing this section, we wish to address the numerous claims made that the kinetic approach is limited in its application to situations where the drag force is linear in the relative velocity between particle and fluid. This is not correct. We refer the reader to section III in 18 on the particle motion which specifically deals with the treatment of non linear drag and how it is used to evaluate the convective and dispersive terms in the kinetic equation. In particular the mean and fluctuating aerodynamic driving force are expressed in terms of the particle mean density weighted particle velocity $\overline{\boldsymbol{v}}(\mathbf{x}, t)$ and incorporated into the particle momentum equations by 
suitably integrating the kinetic equation over all particle velocities. We refer also to [15] where using the kinetic equation for nonlinear drag, an evaluation is made of the long term diffusion coefficient for high inertial particles in homogeneous isotropic statistically stationary turbulence.

\section{SUMMARY AND CONCLUSIONS}

This paper is about well-posedness and realizability of the kinetic equation and its relationship to the GLM equation for modeling the transport of small particles in turbulent gas-flows. Previous analyses [11, 12] claim that the kinetic equation is ill-posed and therefore invalid as a PDF description of dispersed two-phase flows. Specifically, it is asserted that the kinetic equation as given in equation (2) has the properties of a backward heat equation and as a consequence its solutions will in the course of time exhibit finite-time singularities. The justification for this claim is based on an analysis centered around the observation that the phase space diffusion tensor $\boldsymbol{B}$ in equation (2) is not positive-definite but possesses both negative and positive eigenvalues. So we have examined the validity of assumptions that lead to this conclusion and in particular the form of the kinetic equation in a moving frame where the $\operatorname{PDF} \widetilde{p}\left(\widetilde{z}_{1}, \widetilde{z}_{2}, t\right)$ refers to that of transformed variables $\widetilde{z}_{1}, \widetilde{z}_{2}$ measured at time $t$ along the principal axes of $\boldsymbol{B}$ (see equation (6)). Based on the negative diffusion coefficient in the transformed PDF equation (8) for the marginal distribution $\widetilde{p_{r}}\left(\widetilde{z}_{1}, t\right)$, these studies seek to show that this equation (and so also equation $(\sqrt{2})$ )) is ill-posed. However a fundamental error is made by assuming incorrectly that the term $\overline{\mathrm{a}}_{1}$ in equation $(8)$ is wholly convective when in fact it is a density weighted variable which because $\widetilde{z}_{1}$ and $\widetilde{z}_{2}$ for the particle motion are coupled in phase space, means that $\overline{\mathrm{a}}_{1}$ has a gradient diffusive component with a $+v e$ diffusion coefficient which offsets the component in equation (8) with a $-v e$ diffusion coefficient. More particularly, we showed that the solution is a Gaussian distribution with covariances that are the solutions of a set of coupled equations in equations 13, (14). Based on these solutions, the resultant convection-gradient diffusion equation for $\widetilde{p}_{r}\left(\widetilde{z}_{1}, t\right)$ is given by equation 15 with a diffusion coefficient $\widetilde{D}_{1}(t)$ given by the sum of the $+v e$ and -ve contributions defined in equations (16). Using an exponential decaying autocorrelation of the fluid velocity measured along a particle trajectory, we obtained analytic solutions for the +ve and -ve components of $\widetilde{D}_{1}$ which show that the +ve component always outweighs the -ve component and that $\widetilde{D}_{1}$ is crucially always +ve. The corresponding +ve values of $\widetilde{D}_{1}$ are shown in Figure 3 which indicate that $\widetilde{D}_{1}(t)$ approaches an asymptotic value that is independent of the particle response $\tau_{p}$, evident from asymptotic expression given in equation (17). Significantly we were able to show that this was a general result for all realizable forms for the flow velocity autocorrelation along particle trajectories and as a consequence the kinetic equation is not ill-posed.

Finally, in the course of our examination of the analysis of ill-posedness, we pointed out a number of issues with the use of the Feynman-Kac formula (FKF). The application of the FKF to equation $\sqrt{8}$ is problematic because equation (8) is not really a Backward Kolmogorov Equation. Furthermore, the claim that the FKF solution to equation (8) implies that the kinetic equation is only solvable for special initial conditions is erroneous. The FKF employs a terminal condition, and therefore there can be only one possible "initial condition", or else solutions to equation (8) would not be unique.

Another important issue was the claim made in [12] that the kinetic equation can be derived from the GLM PDF equation. That in fact the GLM is a more general approach than the kinetic approach. We showed that this is not the case, that the assumptions made regarding the averaging process lead again to a fundamental error in the closure approximation that negates this claim.

In the final part of our analysis we sought to give a more balanced appraisal of the benefits of both PDF approaches and in particular to point out the limitations of the GLM for gas-particle flows that have previously been ignored. We regarded these limitations to be areas for improvement of the GLM rather than inherent deficiencies. As we pointed out, the value of models of this sort is that features inherent in more fundamental approaches like the kinetic approach can be included in an ad-hoc manner. None the less there were terms that were fundamental to the modeling like the fluctuating convective strain rate contribution which had been ignored but which contained valuable information on the relationships between Lagrangian and Eulerian timescales and the dependence on particle inertia. We suggested how additional features like particle clustering and drift in inhomogeneous turbulent flows particularly in turbulent boundary layers might be included in the model to make it more complete. This is one of the ways that the kinetic approach can support the PDF dynamic model by giving specific formulae for these additional features.
[1] A. Bragg, D C Swailes, and R Skartlien. Drift-free kinetic equations for turbulent dispersion. Phys. Rev. E, 86:056306, 2012a.

[2] S. Chandrasekhar. Stochastic problems in physics and astronomy. Rev. Mod. Phys., 15 (1):1-89, 1943.

[3] S. Chapman and T. G. Cowling. In The Mathematical Theory of Non-uniform Gases. CUP, Cambridge Press, 1952. 
[4] K. F. F. Darbyshire and D. C. Swailes. A pdf model for particle dispersion with stochastic particle-surface interactions, fed-236, gas-solid flows. In FED-236, Gas-Solid Flows, ASME 51-56, pages 51-56. 1996.

[5] Uriel Frisch. Turbulence: The Legacy of A. N. Kolmogorov. CUP, page 87, 1995.

[6] D. C. Haworth and S. B. Pope. A generalized Langevin model for turbulent flow. Phys Fluids, 29:387-405, 1986.

[7] K. E. Hyland, S M ${ }^{\mathrm{c}}$ Kee, and M. W. Reeks. Derivation of a pdf kinetic equation for the transport of particles in turbulent flows. J. Phys. A: Math. Gen., 32:6169-6190, 1999.

[8] R. H. Kraichnan. Lagrangian History Direct Interaction for turbulence. Phys. Fluids, 8:575, 1965.

[9] J. M. MacInnes and F. V. Bracco. Stochastic particle dispersion modelling and the tracer partcle limit. Phys Fluids A, 4(12):2809-2824, 1992.

[10] M. R. Maxey. The gravitational settling of aerosol particles in homogeneous turbulence and random flow fields. J. Fluid Mech., 174:441-465, 1987.

[11] J-P Minier. Statistical descriptions of polydisperse turbulent two-phase flows. Physics Reports, 665:1 - 122, 2016.

[12] J-P. Minier and C. Profeta. Kinetic and dynamic probability-density-function descriptions of disperse turbulent two-phase flows. Phys.Rev. E., 92:053020, 2015.

[13] S. G. Pope. Pdf methods for turbulent reactive flows. Progress in Energy and Combustion Science, page 119, 1991.

[14] M. W. Reeks. On the dispersion of small particles in an isotropic turbulent flow. J. Fluid Mech.., 83:529-546, 1977.

[15] M. W. Reeks. Eulerian direct interaction applied to the statistical motion of particles in a turbulent fluid. $J$. Fluid Mech., 97(3):669-690, 1980.

[16] M. W. Reeks. The transport of discrete particles in inhomogeneous turbulence. J. Aerosol Sci., 14(6):729-739, 1983.

[17] M. W. Reeks. On a kinetic equation for the transport of particles in turbulent flows. Phys. Fluids, 3 (3):446-456, 1991.

[18] M. W. Reeks. On the Continuum Equations for dispersed particles in non uniform flows. Phys. Fluids, 4(6):12901303, 1992.

[19] M. W. Reeks. On probability density function equations for particle dispersion in a uniform shear flow. J. Fluid Mech, 522:263-302, 2005.

[20] M. W. Reeks. On the probability density function equation for particle dispersion in a uniform shear flow. J. Fluid Mech., 522:263-302, 2005.
[21] M. W. Reeks and S. McKee. The importance of Random Galilean Transformation Invariance in modelling dispersed particle flows. In D.Stock et al., editor, GasSolid Flows, volume 121 of ASME-FED, pages 39-43. ASME, 1991.

[22] M. W. Reeks and O. Simonin. Pdf models. In C. T. Crowe, editor, Handbook of Multiphase Flows, pages 1389 - 13-113. CRC Press, 2006.

[23] M. W. Reeks, O. Simonin, and P. Fede. Pdf models for particle transport, mixing and collisions, in Computational Methods pp 144-202. In Multiphase Flow Handbook, 2nd Edition. CRC Press for Taylor Francis Group, 2017.

[24] R. Rzehak and W. Zimmermann. Inertial effects in Brownian motion of a trapped particle in shear flow. Physica A, 324:495-508, 2003.

[25] O. Smonin. Statistical and continuum modelling of turbulent reactive particulate flows part II: Application of a two-phase second-moment transport model. Combustion and Turbulence in Two-Phase Flows. Lecture Series Programme, von Karman Institute for Fluid Dynamics, Belgium, 1996.

[26] D. Swailes and K. Darbyshire. A generalized FokkerPlanck equation for particle transport in random media. Physica A, 42, 1997.

[27] D. Swailes and K. Darbyshire. Probabilistic models for particle and scalar transport in fluctuating flows: An evaluation of simple closure approximations. Physica A, 262:307-327, 1999.

[28] D. C. Swailes, Y. A. Sergeev, and A. Parker. ChapmanEnskog closure approximation in the kinetic theory of dilute turbulent gas-particulate suspensions. Physica A, 254:517-547, 1998.

[29] P. van Dijk and D. C. Swailes. Hermite-DG methods for pdf equations modelling particle transport and deposition in turbulent boundary layers. J. Comp. Phys., 231(14):4094-4920, 2012.

[30] M. R. Wells and D. E. Stock. The effects of crossing trajectories on the dispersion of particles in a turbulent flow. J. Fluid Mech., 136:31-62, 1983.

[31] L. I. Zaichik, V. M. Aliphenkov, and E. G. Sinaiski. In Particles in Turbulent Flows. Wiley-VCH, 2008.

[32] L. I. Zaichik, V. M. Aliphenkov, and E. G. Sinaiski. Chapter 1, sect.1.3. In Particles in Turbulent Flows. Wiley-VCH, 2008.

[33] L. I. Zaichik, V. M. Aliphenkov, and E. G. Sinaiski. Chapter 2, sect. 2.1. In Particles in Turbulent Flows. Wiley-VCH, 2008. 